\title{
Mortality from COVID-19 in Patients with COPD: A US Study in the N3C Data Enclave
}

\section{Daniel Meza \\ Basil Khuder \\ Joseph I Bailey \\ Sharon R Rosenberg \\ Ravi Kalhan \\ Paul A Reyfman (D)}

Northwestern University, Feinberg School of Medicine, Division of Pulmonary and Critical Care Medicine, Chicago, IL, USA
Correspondence: Paul A Reyfman Northwestern University Feinberg School of Medicine, Department of Medicine (Pulmonary and Critical Care Medicine), 240 East Huron St. 2-4I5, Chicago, IL, 606II, USA

Tel +3 I2-503-0325

Email paul.reyfman@northwestern.edu

\section{Introduction}

Coronavirus disease 2019 (COVID-19), caused by severe acute respiratory syndrome coronavirus 2 (SARS-CoV2), emerged in late 2019 leading to a global pandemic. COVID-19, a cause of severe viral pneumonia, has resulted in over 2.6 million deaths worldwide and over 500,000 deaths in the United States as of February 2021., Observational research suggests that the risk of mortality increases with the presence of comorbidities: obesity, hypertension (HTN), diabetes mellitus type 2 (DM), and chronic lung disease. ${ }^{3}$ Over the last 40 years in the United States, chronic obstructive pulmonary disease (COPD) has become the fourth leading cause of death. ${ }^{4}$ Pneumonia is associated with an increased risk of hospitalization, intubation, and mortality in people with COPD. ${ }^{5}$ Patients with COPD may be susceptible to worse outcomes from COVID-19 pneumonia than patients without COPD. ${ }^{6,7}$

Given the increased vulnerability of this population, it is important to understand the risk of COVID-19 related mortality in people with COPD. The National Center for Advancing Translational Sciences (NCATS) established the National COVID Cohort Collaboration (N3C), a partnership among 81 academic hubs to share COVID-19 clinical data from electronic health records as part of a platform for answering critical research questions. ${ }^{8,9}$ The N3C compiles data from patients who were tested for COVID-19, or who were symptomatic and highly suspected to have COVID-19. The data obtained from electronic health records include demographics, symptoms, lab test results, procedures, medications, medical conditions, and physical measurements. The data are placed in a cloud-based research environment maintained by NCATS. We used this novel tool to assess the risk of mortality following COVID-19 diagnosis in patients with COPD compared with patients without COPD.

\section{Methods and Analysis}

We performed a query of the N3C limited data set. Patients were selected for inclusion if they were over the age of 35 and had a positive COVID-19 PCR test. We collected data on demographics, hospitalization, and prior ICD-9/10 diagnoses of COPD, chronic kidney disease (CKD), obesity, DM, and HTN. We used the chi-square test to assess differences in characteristics between patients with and without COPD. We performed multivariable logistic regression, adjusting for age, male sex, DM, HTN, CKD, and obesity to assess the odds of mortality following COVID-19 diagnosis in patients with and without COPD. Statistical analysis was done using the secure NCATS Data Enclave, which utilizes the Palantir platform and resides in Amazon 
Web Services GovCloud. Through Palantir we performed analyses using $\mathrm{R}$ (version 3.5.1). This study was reviewed by the Northwestern University Institutional Review Board (IRB) and received an exemption determination. The requirement for informed consent was waived, as confirmation that the data was anonymized and maintained with confidentiality, and the investigators agreed to make no attempt to identify patients based on the variables in the limited dataset.

\section{Results}

As of February 16, 2021 a total of 3,453,825 patients were part of the $\mathrm{N} 3 \mathrm{C}$ database. Of those, 387,008 patients tested positive for COVID-19 by PCR and 7549 had a diagnosis of COPD (Table 1). The mean age of patients with COPD was 70.5 years versus 57.9 years for patients without COPD. The majority of patients with COPD were white (71\%) followed by African American (19\%). Patients with COPD had higher rates of HTN (74\% vs 39\%), DM (38\% vs $22 \%$ ), obesity (34\% vs $24 \%$ ), and CKD (31\% vs $10 \%)$. Patients with COPD had higher rates of hospital admissions (62\% vs 28\%). The mortality rate of patients with COPD was $15 \%$ compared to $4 \%$ in patients without COPD. Our unadjusted odds ratio of mortality of patients with COPD and COVID-19 diagnosis was 6.19 (95\% CI 5.79-6.62, p-value <0.001). In a multivariable logistic regression analysis the adjusted odds ratio for mortality
[95\% CI] in patients with versus without COPD was 2.1 [1.96, 2.26, p-value $<0.001]$ (Figure 1).

\section{Discussion}

Among patients in the $\mathrm{N} 3 \mathrm{C}$, the odds of mortality from COVID-19 were higher in patients who had COPD compared with those who did not, even after adjusting for other known risk factors. The major strength of this study is the large number of patients included in the analysis. We found that even after adjusting for other comorbidities, the presence of COPD was associated with a higher mortality from COVID-19. Limitations of this retrospective observational study include the potential presence of residual confounders or misclassification of patients with COPD or other comorbidities. We were not able to assess smoking status, a critical potential confounder, because this information was not documented in the majority of patients. Possible mechanisms underlying an increased risk of death from COVID-19 in patients with COPD include a heightened bronchial epithelial cell expression of angiotensin-converting enzyme- 2 (ACE-2) expression, known to be needed for infection. ${ }^{10}$ Other potential factors include worse baseline lung function and lower oxygen levels in patients with COPD. Our findings are consistent with prior work demonstrating worse outcomes from bacterial pneumonia in patients with COPD. ${ }^{5}$ Further prospective research is needed to confirm and elucidate the cause of this association.

Table I Characteristics of Patients with COVID-19 (PCR Positive) in the N3C Data Enclave

\begin{tabular}{|c|c|c|c|}
\hline & COPD $(n=7449)$ & NO COPD $(n=273,963)$ & p-value \\
\hline Age, mean (years) & 70.5 & 57.9 & \\
\hline \multicolumn{4}{|l|}{ Gender } \\
\hline Female, No. (\%) & 3500 (47\%) & 147,639 (54\%) & $<0.001$ \\
\hline Race & & & $<0.001$ \\
\hline Black, No. (\%) & $14 \mid 2$ (19\%) & $47,046(17 \%)$ & \\
\hline White, No. (\%) & 5310 (7I\%) & 174,487 (64\%) & \\
\hline Asian, No. (\%) & $93(1 \%)$ & $6765(2 \%)$ & \\
\hline Hispanic, No. (\%) & $294(4 \%)$ & $32,331(12 \%)$ & $<0.001$ \\
\hline \multicolumn{4}{|l|}{ Comorbidities } \\
\hline Diabetes, No. (\%) & 2849 (38\%) & $59,459(22 \%)$ & $<0.001$ \\
\hline Obesity, No. (\%) & 2539 (34\%) & 64,591 (24\%) & $<0.001$ \\
\hline CKD, No. (\%) & $2328(31 \%)$ & $28,195(10 \%)$ & $<0.001$ \\
\hline HTN, No. (\%) & $554 \mid(74 \%)$ & 108,105 (39\%) & $<0.001$ \\
\hline Hospitalizations, n (\%) & $463 \mid(62 \%)$ & $76,875(28 \%)$ & $<0.001$ \\
\hline Deaths, n (\%) & 1107 (15\%) & 10,126 (4\%) & $<0.001$ \\
\hline
\end{tabular}




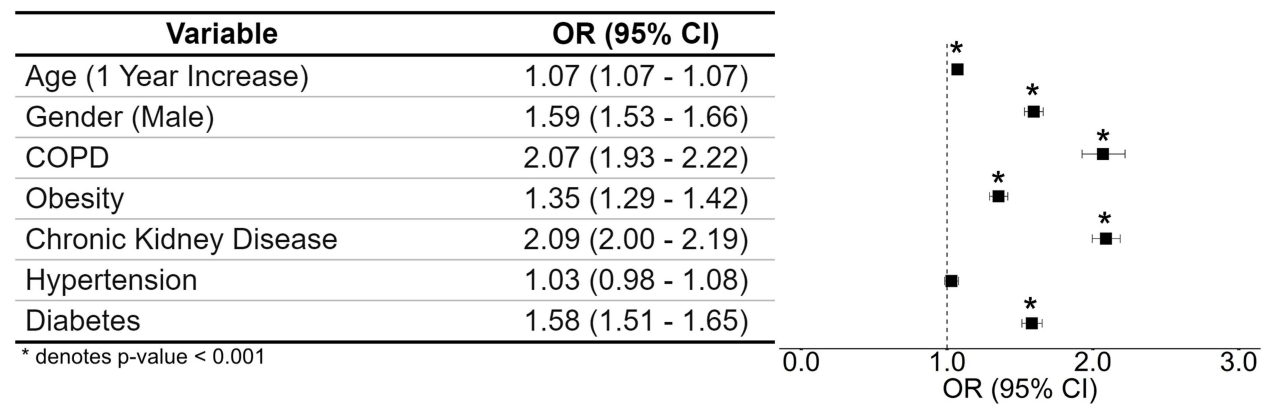

Figure I Multivariable logistic regression analysis of risk factors for death from COVID-19 in the N3C data set. Multivariable logistic regression analysis demonstrated that age, male gender and presence of COPD, obesity, chronic kidney disease, and diabetes were all associated with increased odds of death from COVID-I9 in the N3C data set.

\section{Acknowledgments}

All authors made a significant contribution to the work reported, whether that is in the conception, study design, execution, acquisition of data, analysis and interpretation, or in all these areas; took part in drafting, revising or critically reviewing the article; gave final approval of the version to be published; have agreed on the journal to which the article has been submitted; and agree to be accountable for all aspects of the work.

P.A.R. is supported by NHLBI K08 HL146943, an ATS Foundation/Boehringer Ingelheim Research Fellowship in ATS, and by the Respiratory Health Association.

The analyses described in this publication were conducted with data or tools accessed through the NCATS N3C Data Enclave (ncats.nih.gov/n3c/about) and supported by NCATS U24 TR002306. This research was possible because of the patients whose information is included within the data and the organizations and scientists who have contributed to the on-going development of this community resource: doi.org/10.5281/zenodo.3979622.

\section{Disclosure}

Dr Ravi Kalhan reports personal fees from AstraZeneca, personal fees from GlaxoSmithKline, personal fees from CVS Caremark, outside the submitted work.

Dr Paul A Reyfman reports personal fees from Medscape, personal fees from Guidepoint, and salary from MSD, outside the submitted work. The authors report no other conflicts of interest in this work.

\section{References}

1. Zhu N, Zhang D, Wang W, et al. A novel coronavirus from patients with Pneumonia in China, 2019. $N$ Engl $J$ Med. 2020;382 (8):727-733. doi:10.1056/NEJMoa2001017

2. University CfSSaEaJH. COVID-19 dashboard; 2021. Available from: https://coronavirus.jhu.edu/map.html. Accessed March 22, 2021.

3. Xie J, Tong Z, Guan X, Du B, Qiu H. Clinical characteristics of patients who died of coronavirus disease 2019 in China. JAMA Netw Open. 2020;3(4):e205619. doi:10.1001/jamanetworkopen.2020.5619

4. Tilert T, Dillon C, Paulose-Ram R, Hnizdo E, Doney B. Estimating the U.S. prevalence of chronic obstructive pulmonary disease using pre- and post-bronchodilator spirometry: the National Health And Nutrition Examination Survey (NHANES) 2007-2010. Respir Res. 2013;14:103. doi:10.1186/1465-9921-14-103

5. Restrepo MI, Mortensen EM, Pugh JA, Anzueto A. COPD is associated with increased mortality in patients with community-acquired pneumonia. Eur Respir J. 2006;28(2):346-351. doi:10.1183/ 09031936.06.00131905

6. Leung JM, Niikura M, Yang CWT, Sin DD. COVID-19 and COPD. Eur Respir J. 2020;56(2):2002108. doi:10.1183/13993003.02108-2020

7. Halpin DMG, Criner GJ, Papi A, et al. Global initiative for the diagnosis, management, and prevention of chronic obstructive lung disease. The 2020 GOLD science committee report on COVID-19 and chronic obstructive pulmonary disease. Am J Respir Crit Care Med. 2021;203(1):24-36. doi:10.1164/rccm.202009-3533SO

8. (NCATS). NIoHNNCfATS. National COVID cohort collaborative data enclave repository. U.S. Department of Health and Human Services, National Institutes of Health; 2021. Available from: https://ncats.nih.gov/n3c. Accessed March 22, 2021.

9. Bennett TD, Moffitt RA, Hajagos JG, et al. Clinical Characterization and Prediction of Clinical Severity of SARS-CoV-2 Infection Among US Adults Using Data From the US National COVID Cohort Collaborative. JAMA Netw Open. 2021;4(7):e2116901. doi:10.1001/ jamanetworkopen.2021.16901.

10. Leung JM, Yang CX, Tam A, et al. ACE-2 expression in the small airway epithelia of smokers and COPD patients: implications for COVID-19. Eur Respir J. 2020;55(5):2000688. doi:10.1183/ 13993003.00688-2020 


\section{Publish your work in this journal}

The International Journal of COPD is an international, peer-reviewed journal of therapeutics and pharmacology focusing on concise rapid reporting of clinical studies and reviews in COPD. Special focus is given to the pathophysiological processes underlying the disease, intervention programs, patient focused education, and self management protocols. This journal is indexed on PubMed Central, MedLine and CAS. The manuscript management system is completely online and includes a very quick and fair peer-review system, which is all easy to use. Visit http://www.dovepress.com/testimonials.php to read real quotes from published authors.

Submit your manuscript here: https://www.dovepress.com/international-journal-of-chronic-obstructive-pulmonary-disease-journal 